\title{
THE HUGHES CONJECTURE AND GROUPS WITH ABSOLUTELY REGULAR SUBGROUPS OR ECF-SUBGROUPS
}

\author{
JOSEPH A. GALLIAN
}

\begin{abstract}
Let $G$ be a finite $p$-group and $H_{p}(G)$ the subgroup generated by the elements of $G$ of order different from $p$. Hughes conjectured that if $G>H_{p}(G)>1$, then $\left|G: H_{p}(G)\right|=p$. Although the conjecture is not true for all $G$, it is shown here that if $G$ has a subgroup $L$ such that $\left|L: L^{p}\right| \leq p^{p-r}(r \geq 1)$ and $p^{r} \leq|G: L| \leq$ $p^{r+p}$ or an $E C F$-subgroup $L$ with $\left|G: L_{2}\right| \leq p^{p+2}$, then $G$ satisfies the Hughes conjecture.
\end{abstract}

Let $G$ be a group, $p$ a prime and $H_{p}(G)$ the subgroup of $G$ generated by the elements of order different from $p$. Hughes conjectured that if $G>$ $H_{p}(G)>1$, then $\left|G: H_{p}(G)\right|=p$. The conjecture is known to be true for any $G$ with $p=2[6]$ or $p=3[11]$ and for any $p$ with $G$ finite and not a $p$-group [7]. Although there is a finite 5-group with $\left|G: H_{5}(G)\right|=25$ [12], many authors have given sufficient conditions for the conjecture to hold. We refer the reader to [4] and [5] for summaries of these results.

In an earlier paper [5], the author proved that if $G$ contains a subgroup $L$ such that $\left|L: L^{p}\right| \leq p^{p-r}(r \geq 1)$ and $L$ has index $p^{r}$ or $p^{r+1}$ in $G$ or a normal $E C F$-subgroup $L$ with $\left|G: L_{2}\right| \leq p^{p+2}$, then $G$ satisfies the Hughes conjecture. In this paper these two results and Theorem 3 of [4] are generalized.

For the remainder of the paper $G$ denotes a finite $p$-group. If $G$ has class $c=c(G)$, we use $G=G_{1}>G_{2}>\cdots>G_{c+1}=1$ to denote the lower central series of $G$. Let $G^{p}=\left\langle g^{p} \mid g \in G\right\rangle$. Following Blackburn, we say $G$ is absolutely regular if $\left|G: G^{p}\right| \leq p^{p-1}$ and we call $G$ an $E C F$-group if $\left|G_{i} / G_{i+1}\right|=p$ for $i=2, \ldots, c$ and $\exp \left(G / G_{2}\right)=p$. An ECF-group $G$ with $\left|G: G_{2}\right|=p^{2}$ is called a group of maximal class.

Theorem 1. Suppose $G$ has a subgroup $L$ such that $\left|L: L^{p}\right| \leq p^{p-r}(r$ $\geq 1$ ) and $p^{r} \leq|G: L| \leq p^{r+p}$. Then $G$ satisfies the Hughes conjecture.

Received by the editors March 6, 1974 .

AMS (MOS) subject classifications (1970). Primary 20D15, $20 \mathrm{D} 25$.

Key words and phrases. Finite $p$-groups, $H_{p}$-problem, Hughes conjecture, $E C F$-groups, absolutely regular groups. 
Proof. Suppose $G$ is a minimal counterexample. By the result mentioned in the second paragraph we have $p^{r+2} \leq|G: L| \leq p^{r+p}$. Let $N$ be a central subgroup of $G$ of order $p$. Then $N \leq H_{p}(G)$ so that $H_{p}(G / N) \leq H_{p}(G) / N$ and $\left|G / N: H_{p}(G / N)\right| \geq\left|G: H_{p}(G)\right|>p$. Also $p^{r} \leq|G / N: L N / N| \leq p^{r+p}$ and $\left|L N / N:(L N / N)^{p}\right| \leq\left|L: L^{p}\right| \leq p^{p-r}$. Thus $G / N$ satisfies the hypothesis of the theorem and it follows that $H_{p}(G / N)=1$. Hence $\left|L^{p}\right| \leq p$, so that $|L|$ $\leq p^{p-r+1}$ and $|G| \leq p^{2 p+1}$. But Corollary 3 in [9], the theorem in [10] and Theorem 1 in [4] together show that a counterexample must have order at least $p^{2 p+2}$. This contradiction proves the theorem.

Theorem 2. Suppose $G$ has an ECF-subgroup $L$ such that $\left|G: L_{2}\right| \leq$ $p^{p+2}$. Then $G$ satisfies the Hughes conjecture.

Proof. Suppose $G$ is a counterexample. As mentioned in the proof of Theorem 1 we must have $|G| \geq p^{2 p+2}$. It follows that $c(L)>p$. There exists a subgroup $M$ of $L$ such that $M_{i}=L_{i}$ for all $i \geq 2$ and $\left|M: M_{2}\right|=p^{2}$ [2, p. 65]. Furthermore, $M$ has a subgroup $N$ of index $p$ such that $N^{p}=M_{p}$ [2, p. 69]. It follows that $\left|N: N^{p}\right|=p^{p-1}$ and $p \leq|G: N| \leq p^{p+1}$. This contradicts Theorem 1.

A special case of Theorem 2 is worth stating.

Corollary 1. If $G$ has a subgroup $L$ of index at most $p^{p}$ which is a group of maximal class, then $G$ satisfies the Hughes conjecture.

Corollary 2. Suppose $G$ possesses a subgroup $L$ of index at most $p^{p}$ and there is an integer s satisfying $p^{p} \leq p^{s} \leq|L|$ such that all the normal subgroups of $L$ of order $p^{s}$ are absolutely regular. Then $G$ satisfies the Hughes conjecture.

Proof. A theorem of Blackburn [3, p. 8] shows that either $L$ is absolutely regular or $L$ is a group of maximal class. By Corollary 1, we may assume that $\left|L: L^{p}\right| \leq p^{p-1}$. If $G=L$, then $G$ is regular [8, p. 332] and $G$ satisfies the Hughes conjecture (see [5]). If $L<G$, then $G$ satisfies the hypothesis of Theorem 1 with $r=1$.

A well-known theorem of P. Hall [8, p. 334] says that if $G$ is not regular then $G$ has a normal subgroup of order $p^{p-1}$ and exponent $p$. For groups which do not satisfy the Hughes conjecture much more can be said.

Corollary 3. If $G$ does not satisfy the Hughes conjecture, then for every subgroup $L$ of $G$ of index $p^{p}$ the number of subgroups of $L$ of order 
$p^{p}$ and exponent $p$ is congruent to 1 modulo $p$. Furthermore, every normal subgroup of index $p^{p}$ in $G$ contains a subgroup of order $p^{p}$ and exponent $p$ which is normal in $G$.

Proof. A pair of results of Berkovič [1, pp. 826, 828] shows that the existence of a subgroup $L$ which does not have the required properties implies that $L$ is either absolutely regular or a group of maximal class. Thus, by either Theorem 1 or Theorem 2, we have a contradiction.

Theorem 3 of [4] says that if $|G|=p^{n}$ and for some fixed $r$ with $3 \leq r$ $<n$ all the normal subgroups of $G$ of order $p^{r}$ are generated by two elements, then $G$ satisfies the Hughes conjecture. That theorem was generalized by Theorem 6 of [5] and we conclude this paper with a different generalization.

Theorem 3. Suppose $G$ has a subgroup $L$ of index at most $p^{p+2}$ in $G$ and every normal subgroup of $L$ of order $p^{r}$ has two generators where $r$ is fixed and $p^{3} \leq p^{r}<|L|$. Then $G$ satisfies the Hughes conjecture.

Proof. Suppose $G$ is a counterexample. Then, since $|G| \geq p^{2 p+2}$ (see proof of Theorem 1) and $|G: L| \leq p^{p+2}$ we have $|L| \geq p^{p}$. Also Theorem 6 of [5] shows that $|G: L| \geq p^{2}$. First suppose $p^{r}=|L| / p$ and $|L| \neq 5^{5}$. Since $p \geq 5$, a theorem of Blackburn [3, p. 19] shows that $L$ is metacyclic or $\left|L: L^{p}\right|=p^{3}$. If $L$ is metacyclic, then $\left|L: L^{p}\right| \leq p^{2}[8, \mathrm{p} .337]$ so that in either case we have $\left|L: L^{p}\right| \leq p^{p-2}$ and Theorem 1 shows that we have derived a contradiction. Now suppose $p^{r}=|L| / p$ and $|L|=5^{5}$. If $\left|L^{5}\right|=1$ or 5 , there is a normal subgroup $N$ of $L$ of order 5 such that $L / N$ has exponent 5 . Since every group of order $5^{4}$ has an Abelian subgroup of order $5^{3}$, let $M / N$ be a subgroup of $L / N$ with this property. Then $|M|=5^{4}$ so that $M / N$ is generated by two elements and is elementary Abelian. This is clearly impossible. Thus $\left|L: L^{5}\right| \leq 5^{3}$ and Theorem 1 again yields a contradiction.

Finally, suppose $p^{r}<|L| / p$. Then another theorem of Blackburn [3, p. 16] implies that $L$ is metacyclic or the elements of $L$ of order at most $p$ form a subgroup $E$ of order $p^{3}$ and $L / E$ is cyclic. If the latter is true, then $c(L) \leq 4<p$ so $L$ is regular. Then $\left|L: L^{p}\right|=|E|=p^{3} \leq p^{p-2}[8, \mathrm{p}$. 327]. Thus, in either case, we have $\left|L: L^{p}\right| \leq p^{p-2}$ in contradiction to Theorem 1 .

\section{REFERENCES}

1. Ja. G. Berkovič, A generalization of the theorems of P. Hall and N. Blackburn and their application to nonregular p-groups, Izv. Akad. Nauk SSSR Ser. Mat. 35 (1971), 800-830 = Math. USSR Izv. 5 (1971), 815-844. MR 45 \#3565. 
2. N. Blackburn, On a special class of p-groups, Acta Math. 100 (1958), 4592. MR $21 \# 1349$.

3. - Generalizations of certain elementary theorems on p-groups, Proc. London Math. Soc. (3) 11 (1961), 1-22. MR 23 \#A208.

4. J. A. Gallian, The $H_{p}$-problem for groups with certain central factors cyclic, Proc. Amer. Math. Soc. 42 (1974) 39-41.

5. - On the Hughes conjecture, J. Algebra (to appear).

6. D. R. Hughes, Partial difference sets, Amer. J. Math. 78 (1956), 650-674. MR 18, 921.

7. D. R. Hughes and J. G. Thompson, The $H_{p}$-problem and the structure of $H_{p}$ groups, Pacific J. Math. 9 (1959), 1097-1101. MR 21 \#7248.

8. B. Huppert, Endliche Gruppen. I, Die Grundlehren der math. Wissenschaften, Band 134, Springer-Verlag, Berlin and New York, 1967. MR 37 \#302.

9. I. D. Macdonald, The Hughes problem and others, J. Austral. Math. Soc. 10 (1969), 475-479. MR $40 \# 7353$.

10. Solution of the Hughes problem for finite p-groups of class $2 p-2$, Proc. Amer. Math. Soc. 27 (1971), 39-42. MR 42 \#6113.

11. E. G. Straus and G. Szekeres, On a problem of D. R. Hughes, Proc. Amer. Math. Soc. 9 (1958), 157-158. MR 20 \#73.

12. G. E. Wall, On Hughes' $H_{p}$-problem, Proc. Internat. Conf. Theory of Groups (Canberra, 1965), Gord on and Breach, New York, 1967, pp. 357-362. MR 36 \#2686.

DEPARTMENT OF MATHEMATICS, UNIVERSITY OF MINNESOTA, DULUTH, MINNESOTA 55812 